\title{
ON SCARCITY OF OPERATORS WITH FINITE SPECTRUM
}

\author{
BY BERNARD AUPETIT ${ }^{1}$
}

Communicated by Paul R. Halmos, January 9, 1976

Let $\rho$ denote the spectral radius of an operator; in 1968-1970 Edoardo Vesentini proved

PROPOSITION 1 ([5] AND [6]). If $\lambda \rightarrow f(\lambda)$ is an analytic function mapping a domain in $\mathbf{C}$ into a complex Banach algebra then $\lambda \rightarrow \log \rho(f(\lambda))$ is subharmonic.

From this we got the following generalization of Newburgh's continuity theorem [4], where $\sigma(x)$ is the union of Sp $x$ and its holes.

Proposition 2 (Almost-continuity theorem). If $\lambda \rightarrow f(\lambda)$ is analytic on a domain $D$ containing $z_{0}$ and if $E$ is a subset of $D$, such that $z_{0} \in \bar{E}, E$ is nonsharp at $z_{0}$, then there exists a sequence $\left(\lambda_{n}\right)$ converging to $z_{0}$ with $\lambda_{n} \in E$, $\lambda_{n} \neq z_{0}$, and $\lim _{n \rightarrow \infty} \sigma\left(f\left(\lambda_{n}\right)\right)=\sigma\left(f\left(\lambda_{0}\right)\right)$.

The same statement with the spectrum is false. If $\delta$ is the diameter of the spectrum, we obtained as well

Proposition 3. With the same hypothesis $\lambda \rightarrow \log \delta(f(\lambda))$ is subharmonic.

All of these results and intricate properties of subharmonic functions, capacity and sharp sets, easily found in [1], give the fundamental theorem

THEOREM 1. Either the set of $\lambda$, such that the spectrum of $f(\lambda)$ is finite, is of outer capacity zero, or there exists an integer $n$ such that the spectrum of $f(\lambda)$ has exactly $n$ elements, for every $\lambda$, except on a closed set of capacity zero, where the spectrum has at most $n-1$ elements.

Kaplansky [3], in 1954, and Hirschfeld-Johnson [2] , in 1972, proved that $A / \operatorname{Rad} A$ is finite dimensional, for a complex Banach algebra $A$, if the spectrum of every element of this algebra is finite. Unfortunately the method does not work for local and real cases. Other persons (Behncke, Wong) obtained the same result for $A^{*}$-algebras supposing the spectrum finite for hermitian elements.

Theorem 1 can be used to get

AMS (MOS) subject classifications (1970). Primary 46H20, 46K99; Secondary 47A10.

1 The author is supported by National Research Council of Canada (Grant A7668).

Copyright $\odot 1976$, American Mathematical Society 
THEOREM 2. Let $A$ be a complex Banach algebra, $H$ a closed real subspace of $A$ such that $A=H+i H$. If there exists a nonempty open set $U$ of $H$ such that $x \in U$ implies $\operatorname{Sp} x$ finite, then $A / \operatorname{Rad} A$ is finite dimensional.

And particularly

THEOREM 3. Let $A$ be a real Banach algebra containing a nonempty open set $U$ such that $x \in U$ implies $\operatorname{Sp} x$ finite; then $A / \operatorname{Rad} A$ is finite dimensional.

THEOREM 4. Let $A$ be a complex Banach algebra with involution such that the set of hermitian elements contains a nonempty open set $U$ with the property $x \in U$ implies $\operatorname{Sp} x$ finite; then $A / \operatorname{Rad} A$ is finite dimensional.

Full details will appear elsewhere.

\section{REFERENCES}

1. M. Brelot, Eléments de la théorie classique du potentiel, Centre de Documentation Universitaire, Paris, $3^{e}$ édition, 1965. MR 21 \#5099.

2. R. A. Hirschfeld and B. E. Johnson, Spectral characterization of finite-dimensional algebras, Nederl. Akad. Wetensch. Proc. Ser A 75 = Indag. Math. 34 (1972), 19-23. MR 46 \#666.

3. I. Kaplansky, Ring isomorphisms of Banach algebras, Canad. J. Math. 6 (1954), 374-381. MR 16, 49.

4. J. D. Newburgh, The variation of spectra, Duke Math. J. 18 (1951), 165-176. MR 14, 481 .

5. E. Vesentini, On the subharmonicity of the spectral radius, Boll. Un. Mat. Ital. (4) 1 (1968), 427-429. MR 39 \#6080.

6. - Maximum theorems for spectra, Essays on Topology and Related Topics (Mémoires dédiés à Georges de Rham), Springer-Verlag, New York, 1970, pp. 111-117. MR 42 \#6612. CANADA

DÉPARTMENT DE MATHÉMATIQUES, UNIVERSITÉ LAVAL, QUÉBEC, 